\title{
Effects of Hyperbaric Oxygen on Skin Blood Flow and Tissue Morphology Following SCIatic Nerve Constriction
}

\author{
George Mychaskiw II, DO, Jun Pan, MD, Sanjay Shah, MD, Alexander Y. Zubkov, MD, Ben Clower, PhD,
} Ahmed E. Badr, MD, and John H. Zhang, PhD

Background: Constriction of the sciatic nerve by loose ligation produces an inflammatory neuropathic injury. This represents an animal model for peripheral mononeuropathy. Oxygen-derived free radicals are suspected to play an important role in the pathogenesis of ischemia/reperfusion injury, leading to neurogenic inflammation. Hyperbaric oxygen (HBO) has been used anecdotally to treat clinically similar conditions in humans, but specific effects on the animal model have not been well studied.

Objective: This study in a rat model examined the effects of hyperbaric oxygen on skin blood flow and tissue morphology by light and electron microscopy following sciatic nerve constriction.

Design: A scientific investigation in a rat model.

Methods: In this study, the neuropathic injury was established by loose ligation of the rat sciatic nerve. The animals were divided into three groups, sham (S, $\mathrm{n}=8$ ), ligation but no treatment $(\mathrm{LN}, \mathrm{n}=8)$ and ligation and

Hyperbaric oxygen therapy is the administration of oxygen under greater than atmospheric pressure to address a wide variety of medical and surgical problems, including complications of uncontrolled decompression during diving, carbon monoxide intoxication, delayed wound

From: Departments of Anesthesiology, Anatomy and Neurosurgery, University of Mississippi School of Medicine, Jackson, MS; and Department of Neurosurgery, Loma Linda University School of Medicine, Loma Linda, CA.

Address Correspondence:

George Mychaskiw II, DO, 2500 North State Street, Jackson, MS 39216-4505

Grants: John H. Zhang, PhD, is the recipient of an American Heart Grant in Aid.

Conflict of Interest: None

Acknowledgement:

Manuscript received on 2/4/2005

Revision submitted on 3/15/2005

Accepted for publication on $3 / 18 / 2005$ treatment with hyperbaric oxygen ( $\mathrm{LT}, \mathrm{n}=8$ ). The treatment group $(n=8)$ received hyperbaric oxygen treatment immediately following the injury and daily for four additional days at the same time interval. One hundred percent $\mathrm{O}_{2}$ at 3 atmospheres absolute pressure (66 feet sea water) was administered for two hours. The hindpaws of the rats were observed by light microscopy, electron microscopy, laser Doppler flowmetry (LDF), and clinically for the presence of edema.

Results: Untreated animals demonstrated marked tissue edema following sciatic constriction, whereas animals that received hyperbaric oxygen had minimal to no edema. The sham group demonstrated normal histology. The group not treated with hyperbaric oxygen demonstrated swollen mitochondria (2-3 times), with loss of cellular integrity, multiple vacuole formation in both nerve and muscle tissue, widened sarcomeres in muscle, and degenerative chang es in the nerve myelin sheaths. The group treated with hyperbaric oxygen demonstrat-

ed preservation of cellular structure including mitochondrial integrity, no vacuole formation, and maintenance of normal, easily identifiable nerve structure.

The sham group had no change of skin blood flow. Skin blood flow of LT group was decreased immediately after ligation $(p<0.05)$ and recovered to baseline level before ligation on Day 5 after four hyperbaric oxygen treatments. Skin blood flow of LN group was decreased immediately after ligation $(p<0.01)$ and did not recover $(p<0.01)$.

Conclusion: This study evaluated tissue changes after nerve injury caused by loose ligation of the sciatic nerve in rats. Hyperbaric oxygen treatment following sciatic nerve injury reduced tissue edema, improved skin blood flow, and preserved muscle and neuronal ultrastructural integrity.

Keywords: Hyperbaric oxygen, nerve chronic constriction injury, sciatic nerve ligation, neuropathic pain, skin blood flow, animal models

healing, and soft-tissue infections (1). Hyperbaric oxygen is believed to suppress free radical formation and oxygen-derived free radicals have been suspected in the development of neurogenic inflammation following injury (2). Little is known about the possible role of hyperbaric oxygen in common investigational models of neuropathic injury. There is a case report of improved signs and symptoms of Complex Regional Pain Syndrome II in a patient undergoing hyperbaric oxygen treatment for carbon monoxide poisoning (3). Hyperbaric oxygen has been anecdotally reported to be effective in the management of peripheral neuropathies, but has not been prospectively evaluated. This study examined the effects of hyperbaric oxygen on a constriction model of nerve injury caused by loose ligation of the rat sciatic nerve (4). In this study we examined whether a short course of hyperbaric oxygen in the acute period following nerve constriction could ameliorate some of the pathologic features of neuropathic injury, including changes in skin blood flow, tissue histology, and edema formation.

\section{Methods}

\section{Animal Model}

A loose ligation model of the rat sciatic nerve, as described by Bennett and Xie (4) was used to simulate an acute neuropathic injury. This model has been well established in our laboratory and consistently produces neuropathic inflammation and allodynia. Allodynia was assumed to occur in this investigation, but was not specifically quantitated. The animal protocol was reviewed and approved by the institutional animal use commit- 
tee, in accordance with NIH/NPS guidelines. Twenty-four adult male SpragueDawley rats (250-350 g) were used in this study. The animals were divided into three groups, sham $(\mathrm{S}, \mathrm{n}=8)$, ligation, no treatment $(\mathrm{LN}, \mathrm{n}=8)$ and ligation and treatment with hyperbaric oxygen (LT, $\mathrm{n}=8$ ).

Animals were anesthetized with $100 \mathrm{mg} / \mathrm{kg}$ Ketamine (Burroughs-Wellcome, USA) and $20 \mathrm{mg} / \mathrm{kg}$ Xylasine (Burroughs-Wellcome, USA) by intraperitoneal injection using aseptic technique and supplemented as necessary. In the ligation groups, the common sciatic nerve on the right side was exposed at the level of the middle thigh by blunt dissection through the biceps femoris. After identification, the common sciatic nerve, just proximal to its trifurcation into the peroneal, sural and tibial nerves, was loosely ligated twice, each ligature $1 \mathrm{~mm}$ apart, using 4-0 chromic catgut (Ethicon, USA), under $25 \mathrm{X}$ magnification. Care was taken to tie the ligatures so that the diameter of the nerve was seen to be just barely constricted. The desired degree of constriction retarded, but did not arrest, circulation through the superficial epineurial vasculature and sometimes produced a small, brief twitch in the surrounding muscle. The incision was closed in layers with 2-0 silk (Ethicon, USA). In the control group, the common sciatic nerve on the right was dissected and isolated at the middle-thigh level and the wound was closed without further manipulation of the nerve. In every animal, an identical dissection was performed on the opposite side, except that the sciatic nerve was not ligated. Postoperatively, the animals were housed in groups of two or three in clear plastic cages with solid floors covered by sawdust. Cages with wire mesh floors were avoided on the assumption that they would exacerbate discomfort arising from the affected hindpaw.

\section{Clinical Observation}

The animals were examined twice daily. During examination, each rat was placed on a table and closely observed by a blinded investigator. The presence of tissue edema was recorded on a scale of 0 to 4 , with 0 being no edema and 4 being marked, severe edema resulting in limb immobility.

\section{Skin Blood Flow Measurement}

Laser Doppler Flowmetry was used to measure skin blood flow (5). Measure- ments were performed by Perimed PF3 with standard $90^{\circ}$ angled probe (Perimed, Linkoping, Sweden) with a duration of two seconds within the frequency between 0.07 to $12 \mathrm{kHz}$. In all three groups, skin blood flow was measured on both of the hindpaws while left hindpaws served as self negative controls after anesthesia but before the surgery, immediately after the surgery, and on Day 5 following induction of anesthesia. The ambient temperature was maintained at $23-25^{\circ} \mathrm{C}$. Skin blood flow measurements were performed with the animal placed in the prone position on a height adjustable plastic floor. The plantar surface of the right or left hindpaw was secured in the desired position with adhesive tape. Laser Doppler Flowmetry measurements were performed within a standardized skin area, consisting of a prominence located at the lateral side of the plantar surface of the hindpaw. The skin area under investigation is innervated by the tibial nerve, which originates from the sciatic nerve (6). Within this specific area, the probe was moved about using a micromanipulator close to the skin surface in order to find the site with maximal skin blood flow. After signal stabilization for at least three minutes, skin blood flow was assessed for three additional minutes. The analog output of the laser Doppler system was digitized by an analog-digital converter and the readings were recorded.

\section{Hyperbaric Oxygen Treatment}

The treatment group $(n=8)$ received hyperbaric oxygen treatment immediately following the injury and daily for four additional days at the same time interval. One hundred percent $\mathrm{O}_{2}$ at 3 atmospheres absolute pressure (66 feet sea water) was used for two hours. Ambient temperature was maintained at $23-25{ }^{\circ} \mathrm{C}$ during the treatments.

\section{Histology}

On Day 5, 24 hours after the last treatment with hyperbaric oxygen and following skin blood flow measurement under anesthesia, the animals were sacrificed by draining whole body blood with infusion of $0.9 \%$ saline via the inferior vena cava through an open sternotomy. After venous drainage became clear, animals were perfused with $2 \%$ buffered glutaraldehyde (0.4-0.5 ml/kg). Samples were taken from muscle and nerve distal to the ligation, preserved in $2 \%$ buffered glutaraldehyde, and prepared for light and elec- tron microscopy evaluation.

\section{Statistical Methods}

Data were stored in an Excel spreadsheet file then analyzed by Prism Graph Pad software (version 4.0). Data collected from right hindpaws among control, ligation without treatment, and ligation with treatment, were analyzed by one way ANOVA. Data collected from the same groups of animals at three different time points were analyzed by one way ANOVA. Data collected from right and left hindpaws were analyzed using a two-tail paired t test. $\mathrm{P}<0.05$ was considered statistically significant.

\section{RESULTS}

\section{Clinical Observation}

The sham group demonstrated no changes in hindpaws. The group treated with hyperbaric oxygen had 0-1+ edema on the right side, which subsided to no edema from Day 2 onwards, whereas the nontreated group had 2-3+ edema on the right side, which remained at the $1-2+$ level after Day 5.

\section{Microscopy}

As shown in figures 1 and 2, light microscopy did not reveal any changes in any groups in nerve or muscle. Electron microscopy at 16,000-80,000 magnification provided the following information:

- The sham group had normal ultrastructural histology.

- The group not treated with hyperbaric oxygen demonstrated swollen mitochondria with loss of cellular integrity, vacuole formation in both nerve and muscle, widened sarcomeres in muscle, and degenerative changes in myelin nerve sheaths.

- The group treated with hyperbaric oxygen demonstrated preservation of cellular structure, including mitochondrial integrity, no vacuole formation, and preservation of normal, easily identifiable nerve structure.

- A blinded observer evaluated electron micrographs of sarcomeres at $16,000 \mathrm{X}$ and neurons at 40,000X and graded cellular changes on a 0 to 3 scale, with 0 being normal histology and 3 being complete disruption of cellular structure and organelles.

- The groups with hyperbaric oxygen could not be distinguished from normal controls, whereas the untreated 


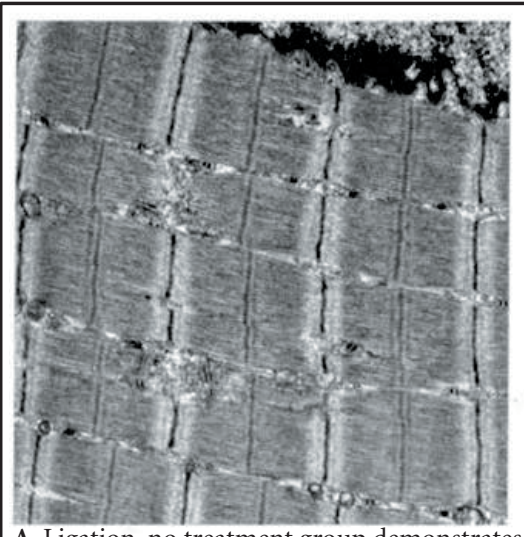

A. Ligation, no treatment group demonstrates marked widening of sarcomeres and mitochondrial swelling, grade 2

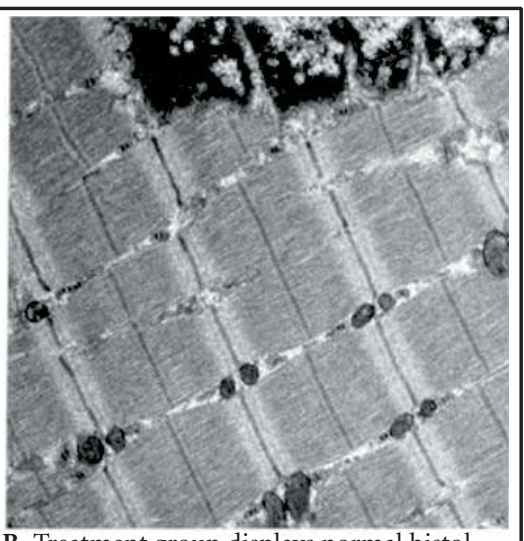

B. Treatment group displays normal histology, indistinguishable from control.

Fig 1. 16,000X electron microscopy of sarcomeres.

groups were graded consistently at 2, indicating mitochondria swollen 2 to 3 times normal size, multiple vacuole formation $(>5)$ and scattered, mild disruption of cell membranes.

\section{Skin Blood Flow}

As shown in Figure 3, in the sham surgery group, there was no significant difference among the skin blood flow results at preligation, immediately postligation, and on Day 5 (one-way ANOVA). There was also no statistically significant difference between left and right hindpaws (paired two-tail t-test). Sham surgery had no effect on skin blood flow.

In the ligation, no treatment group, skin blood flow of right hindpaws decreased immediately following surgery and on Day 5, compared to preligation

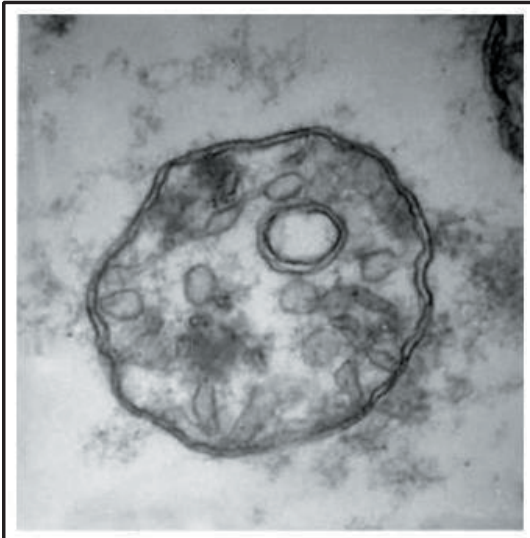

A. Ligation, no treatment group demonstrates vacuole formation, mitochondrial swelling and scattered disruption of cellular structures, grade 2. data $(p<0.01$, one-way ANOVA). Compared to the left hindpaws (self negative control), skin blood flow of the right hindpaws demonstrated no significant difference before ligation (paired two-tail t-test), but decreased significantly immediately postligation and on Day $5(\mathrm{p}<0.01$, $\mathrm{p}<0.05$, respectively, two-tail t-test).

In the ligation with hyperbaric oxygen treatment group, the skin blood flow of the right hind paws decreased significantly immediately after ligation $(\mathrm{p}<0.05$, one-way ANOVA). On Day 5, after four days of treatment with hyperbaric oxygen, skin blood flow recovered to at or near pre-injury levels in the treated group, while skin blood flow in the nontreated group remained significantly depressed $(\mathrm{p}<0.01)$.

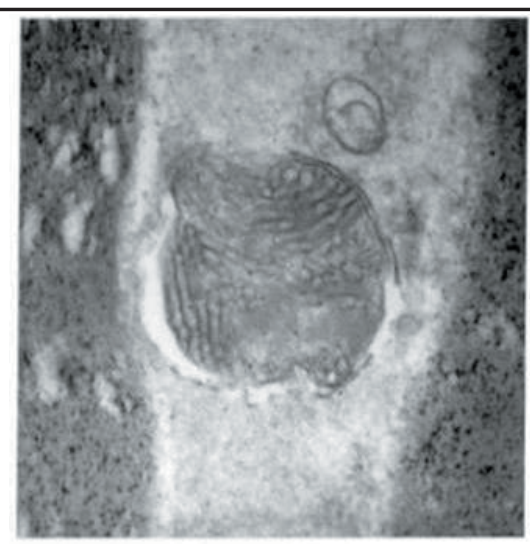

B. Treatment group displays normal histology, indistinguishable from control.

\section{DISCUSSION}

In our study, a peripheral mononeuropathy model was produced in adult male Sprague-Dawley rats by placing loosely constrictive ligatures around the common sciatic nerve. This resulted in alterations of skin blood flow, tissue edema and histology, similar to changes that accompany peripheral neuropathies in humans (4).

Our study confirms the hypothesis of neurogenic inflammation by loose ligation of sciatic nerve, as all the changes were observed distal to the point of ligation. Studies have demonstrated involvement of primary afferent nociceptive c-fibers in an afferent orthodromic and efferent antidromic manner. Consequent nociceptive impulses and neuropeptides released from nerve endings lead to reduction in skin blood flow, collectively leading to ischemia, necrosis and increased vascular permeability in skeletal muscle and nerve, a phenomenon referred to as neurogenic inflammation. In our study, neurogenic inflammation was identified by clinical observation of edema of the extremity distal to nerve ligation and measurement of skin blood flow before, and five days after, ligation.

Hyperbaric oxygen therapy involves inhalation of $100 \%$ oxygen under a pressure greater than 1 atmosphere absolute (ATA) (7). This has been used to treat acute conditions (decompression sickness, air embolism, carbon monoxide poisoning, clostridial myonecrosis, acute traumatic ischemia) as well as chronic conditions (irradiated tissue necrosis, problem wounds). The therapeutic effects of hyperbaric oxygen result from two features of treatment: mechanical effects of increased pressure and physiologic effects of hyperoxia. At normal atmospheric pressure, the oxygen in arterial blood is almost entirely bound to hemoglobin. However, while breathing 100\% oxygen at 2 to 3 ATA, a significant proportion of arterial oxygen is in the dissolved form. Despite some reduction in blood flow during exposure to hyperbaric oxygen, an increase in tissue oxygenation can be observed in mixed venous, transcutaneous, and tissue measurements (8). Neutrophils play an important role as the prime culprit in neurogenic inflammation, as they adhere to the walls of vessels and release proteases, producing free radicals, leading to pathologic tissue destruction (9). Hyperbaric oxygen can inhibit

Fig. 2. 40,000X electron microscopy of neurons. 


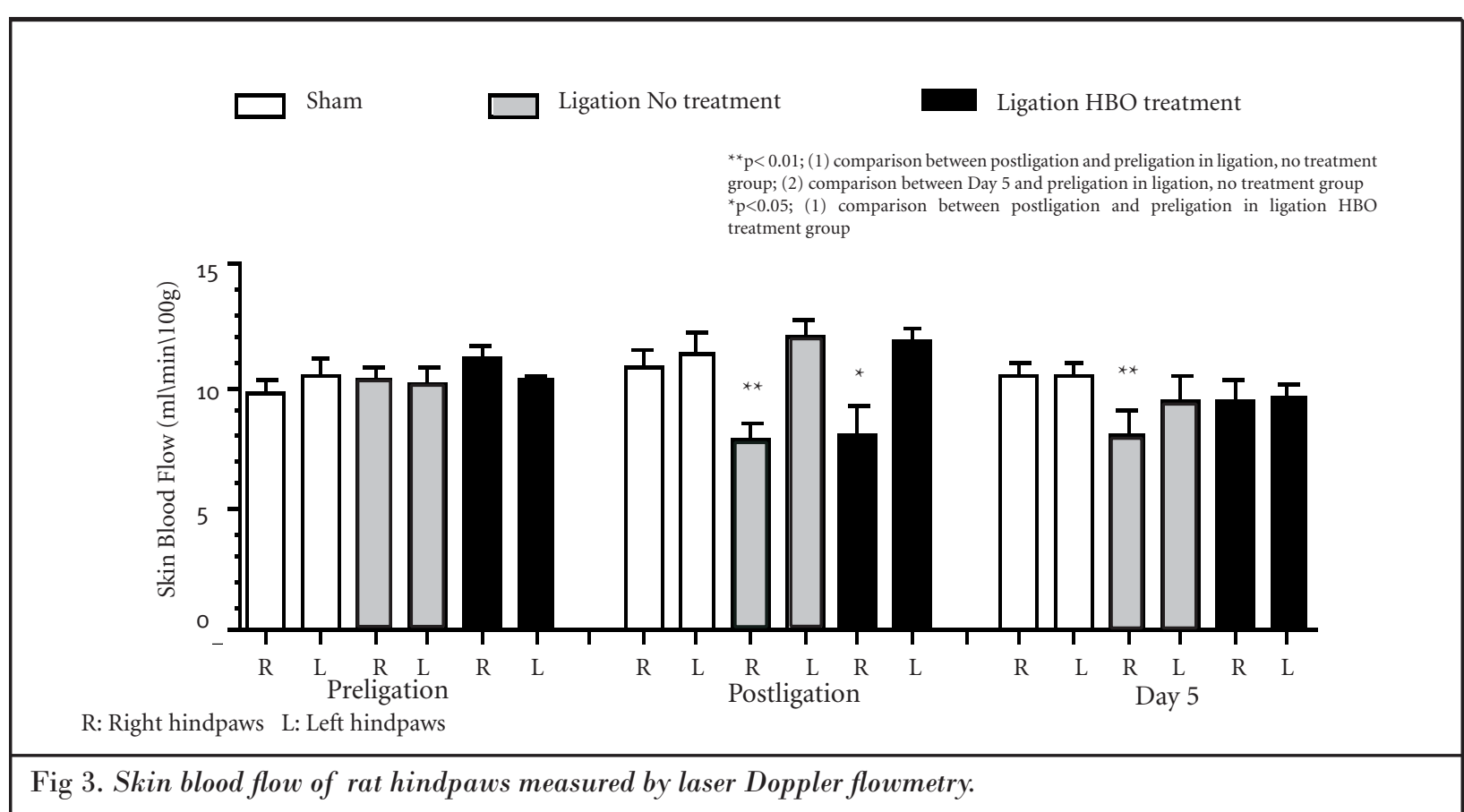

neutrophil adherence and tissue destruction $(10,11)$.

In this study, skin blood flow decreased after nerve ligation in all animals and improved in the treated group, which negated any possibility of accidental vascular ligation causing hypoxic or anoxic cellular death. Although neurogenic inflammation may have resulted from exposure of the nerve itself, the sham group did not demonstrate any changes in skin blood flow or cellular structure. This study supports the role of oxygen-derived free radicals and ischemia/reperfusion injury in the pathogenesis of neuropathic injury and suggests that hyperbaric oxygen can reverse or prevent tissue damage and improve skin blood flow.

\section{ConcLusion}

This study demonstrated a beneficial role of hyperbaric oxygen in ameliorating muscle and neuronal injury in a sciatic nerve ligation model in rats. Hyperbaric oxygen treatment following sciatic nerve injury reduced tissue edema, improved skin blood flow, and preserved muscle and neuronal ultrastructural integrity. Further study is required to evaluate the potential role of hyperbaric oxygen as a therapy for neurogenic inflammation following nerve injury.

\section{ACKNOWLEDGMENTS}

The authors thank Dr. Glenn Hoskins for electron microscopic pictures and Ms.

\begin{tabular}{l}
\hline AUTHOR \\
\hline George Mychaskiw II, DO \\
Professor \\
Department of Anesthesiology \\
Univ. of Mississippi Medical Center, \\
2500 North State Street \\
Jackson, MS 39216-4505 \\
E-mail: gmychaskiw@anesthesia.ums \\
med.edu
\end{tabular}
med.edu

\section{Ahmed E. Badr, MD}

Associate Professor

Department of Anesthesiology

Univ. of Mississippi Medical Center

2500 North State Street

Jackson, MS 39216-4505

\section{Ben R. Clower, PhD}

Professor, Dept. of Anatomy

Univ. of Mississippi Medical Center

2500 North State Street

Jackson, MS 39216-4505

\section{Jun Pan, MD}

Instructor, Dept. of Anesthesiology Univ. of Mississippi Medical Center 2500 North State Street

Jackson, MS 39216-4505
Amanda Moncrief for her support in the neurosurgery research laboratory.

\section{Author Affiliation:}

Sanjay Shah, MD

Resident

Department of Anesthesiology Univ. of Mississippi Medical Center, 2500 North State Street Jackson, MS 39216-4505

Alexander Y. Zubkov, MD Resident

Department of Neurosurgery University of Mississippi Medical Center, 2500 North State Street Jackson, MS 39216-4505

\section{John H. Zhang, PhD}

Professor of Anesthesiology and Neurosurgery

Department of Neurosurgery Loma Linda University School of Medicine

12234 Anderson St.

Loma Linda, CA 92354 


\section{REFERENCES}

1. Sheridan RL, Shank ES. Hyperbaric oxygen treatment: a brief overview of a controversial topic. J Trauma 1999; 47:426-435.

2. Buras J. Basic mechanisms of hyperbaric oxygen in the treatment of ischemia-reperfusion injury. Int Anesthesiol Clin 2000; 38:91-109.

3. Peach $\mathrm{G}$. Hyperbaric oxygen and the reflex sympathetic dystrophy syndrome: a case report. Undersea Hyperb Med 1995; 22: 407-408.

4. Bennett GJ, Xie YK. A peripheral mononeuropathy in rat that produces disorders of pain sensation like those seen in man. Pain 1988; 33:87-107.
5. Daemen MA, Kurvers HA, Kitslaar PJ, Slaaf DW, Bullens PH, Van den Wildenberg FA. Neurogenic inflammation in an animal model of neuropathic pain. Neurol Res 1998; 20:41-45.

6. Swett JE, Woolf CJ. The somatotopic organization of primary afferent terminals in the superficial laminae of the dorsal horn of the rat spinal cord. J Comp Neurol 1985; 231:66-77.

7. Grim PS, Gottlieb LJ, Boddie A, Batson E. Hyperbaric oxygen therapy. JAMA 1990; 263:2216-20.

8. Moon RE. Use of hyperbaric oxygen in the management of selected wounds. Adv Wound Care 1998; 11:332-334.
9. Weiss SJ. Tissue destruction by neutrophils. N Engl J Med 1989; 320:365-376.

10. Zamboni WA, Roth AC, Russell RC, Nemiroff PM, Casas L, Smoot EC. The effect of acute hyperbaric oxygen therapy on axial pattern skin flap survival when administered during and after total ischemia. / Reconstr Microsurg 1989; 5:343-350.

11. Zamboni WA, Roth AC, Russell RC, Graham B, Suchy H, Kucan JO. Morphologic analysis of the microcirculation during reperfusion of ischemic skeletal muscle and the effect of hyperbaric oxygen. Plast Reconstr Surg 1993; 91:1110-1123. 